\title{
DERECHO E INTERNET: INTRODUCCIÓN A UN CAMPO EMERGENTE PARA LA INVESTIGACIÓN Y PRÁCTICA JURÍDICAS*
}

\author{
William Guillermo Jiménez * \\ Orlando Meneses Quintana***
}

Fecha de recepción: 12 de mayo de 2016

Fecha de evaluación: 11 de noviembre de 2016

Fecha de aprobación: 21 de marzo de 2017

Artículo de reflexión

DOI: http://dx.doi.org/10.18359/prole.3040

Forma de citación: Jiménez, W. G. \& Meneses, O. (2017). Derecho e Internet: introducción a un campo emergente para la investigación y práctica jurídicas. Revista Prolegómenos Derechos y Valores, 20, 40, 43-61. DOI: http://dx.doi.org/10.18359/prole.3040

\section{Resumen}

El desarrollo de las TIC e Internet representan un reto para los tradicionales límites del derecho afianzados en la noción de soberanía y la localización espacial de las actividades. Las características del Internet tales como su naturaleza transfronteriza, independencia geográfica, amplia cobertura, anonimato, portabilidad, fácil reproducción, convergencia, conectividad y difícil control, son desafíos para el nuevo derecho. El objetivo es presentar un panorama sobre los aspectos más relevantes que Internet trae para el derecho, así como las áreas o temáticas más críticas o controversiales; mediante un método cualitativo e inductivo, usando fuentes primarias y secundarias y técnicas de revisión documental, estudios de caso y observación directa. Se concluye que es necesario que académicos, jueces y abogados, conozcan más los aspectos tecnológicos de la comunicación virtual, para así entender, manejar y resolver los problemas jurídicos que se presentan en el ambiente de Internet.

\section{Palabras clave:}

Derecho de Internet, tecnología y derecho, tecnologías de la información, derecho informático.

\footnotetext{
Producto de investigación del proyecto: "Derecho e Internet. Regulación jurídica y jurisdicción en los casos de derecho a la intimidad vs. Derecho, libertad de expresión y seguridad", grupos de investigación "Teoría del Derecho, de la Justicia y de la Política" de la Universidad La Gran Colombia (Bogotá, Colombia) y "Política, Derecho y Gestión Pública" de la Escuela Superior de Administración Pública (Bogotá, Colombia).

* Abogado y administrador público. Ph.D. en Derecho (Vrije Universiteit Amsterdam, Ámsterdam, Países Bajos), Ph.D. en Ciencia Política (Universidad de Santiago de Compostela, Santiago de Compostela, España), especialista en Desarrollo Regional, especialista en Derecho Administrativo. Profesor titular Escuela Superior de Administración Pública (Bogotá, Colombia). Abogado independiente. Correo electrónico: willjime@esap.edu.co

Sociólogo, magíster en Ética y Política (Universidad Nacional de Colombia, Bogotá, Colombia). Vinculado al Centro de Investigaciones de la Universidad La Gran Colombia (Bogotá, Colombia). Correo electrónico: orlando.mesenes@ugc.edu.co
} 


\title{
LAW AND INTERNET: INTRODUCTION TO AN EMERGENT FIELD FOR BOTH LEGAL RESEARCH AND PRACTICE
}

\begin{abstract}
Summary
The ICT and the Internet development represent a challenge to the traditional law borders, which are strengthened on the notion of sovereignty and the spatial localization of activities. Features of the internet such as its cross-border nature, geographic independence, broad coverage, anonymity, portability, easy reproduction, convergence, connectivity and difficulty to control, are emerging challenges to the new law. The objective is to present a panoramic view over the most relevant aspects brought by the internet to law, as well as the most controversial or critical topics or fields through a qualitative and inductive method, using both primary and secondary sources and techniques for documentary review, case study, and direct observation. It is concluded the need for scholars, judges and attorneys to familiarize themselves with the technological aspects of virtual communication to understand, manage and resolve the legal concerns found within the internet.
\end{abstract}

\section{Keywords:}

Internet Law; technology and law; information technology, computer law.

\section{DIREITO E INTERNET: INTRODUÇÃO A UM CAMPO EMERGENTE PARA A PESQUISA E A PRÁTICA JURÍDICAS}

\begin{abstract}
Resumo
O desenvolvimento das TIC's e da Internet representa um desafio para os limites tradicionais do direito já estabelecido na noção de soberania e a localização espacial das atividades. As características da Internet, tais como a sua natureza transfronteiriça, independência geográfica, sua extensa cobertura, seu anonimato, sua portabilidade, fácil reprodução, convergência, conectividade e difícil controle, são desafios para o novo direito. O objetivo é apresentar uma visão geral sobre os aspectos mais importantes que a Internet traz para o direito, bem como as áreas ou temáticas mais críticas ou controversas; através de um método qualitativo e indutivo, usando fontes primárias e secundárias e técnicas de analise documental, estudos de caso e observação direta. Conclui-se que é necessário que acadêmicos, juízes e advogados, conheçam mais os aspectos tecnológicos da comunicação virtual, a fim de compreender, lidar e resolver os problemas jurídicos que possam surgir no ambiente da Internet.
\end{abstract}

\section{Palavras-chave:}

Direito da Internet; tecnologia e direito; tecnologias da informação, direito informático.

\section{Introducción: la Internet y las tecnologías de la información y la comunicación}

El aparecimiento y desarrollo de las tecnologías de la información y la comunicación (TIC) están transformado dramáticamente los procesos productivos y laborales, la manera de hacer negocios, los trámites y servicios públicos, los métodos de enseñanza-aprendizaje, los hábitos de diversión y entretenimiento y en fin, las diversas formas de interacción humana. Lo anterior representa 
todo un desafío para las diferentes áreas del conocimiento y el desempeño profesional, en las cuales el derecho no es la excepción.

Las TIC son un rasgo distintivo de la actual sociedad del conocimiento y de la información, y una herramienta poderosa en el proceso de globalización. Se puede decir que las TIC están integradas por un conjunto de conceptos, soportes, canales, programas y dispositivos que almacenan, procesan, recuperan y transmiten información de forma veloz y de variadas maneras. Se pueden definir como: "el conjunto de procesos y productos derivados de las nuevas herramientas (hardware y software), soportes de la información y canales de comunicación relacionados con el almacenamiento, el procesamiento y la transmisión digital de la información" (Guerra, 2012, p. 13).

Las TIC se pueden clasificar según variados criterios, aquí se escogen cuatro: redes, terminales, aplicativos/software y servicios. En cuanto a redes, se citan las más importantes: telefonía fija, banda ancha e Internet, telefonía móvil (celular), redes de televisión, redes en el hogar (WI-FI $)^{1}$, intranet, redes LAN/WAN ${ }^{2}$ y por supuesto, las redes sociales (Facebook, YouTube, Twitter, Instagram, Linkedln, MySpace, etc.).

Respecto de los terminales o puntos de acceso a la información están conformados por una gran variedad de dispositivos como los servidores, las computadoras personales (PC), los computadores portátiles (laptop), las tabletas, los teléfonos celulares (de amplia variedad, incluyendo los smartpho-

$1 \quad$ Iniciales de la expresión wireless fidelity (fidelidad inalámbrica).

2 LAN (local area network) es una red corporativa instalada en una misma oficina, edificio o sedes de la empresa, que facilita intercambiar información y compartir recursos desde los diferentes puestos de trabajo; por ejemplo, cuando recibo un correo interno o cuando envío una impresión desde mi computador a una impresora compartida. WAN (wide area network) es una red que interconecta varias LAN mediante encaminadores o routers, pudiendo cubrir ciudades, regiones, países e incluso continentes. nes), el telefax, las impresoras y escáneres, los televisores (LCD, plasma, led), los reproductores portátiles de audio y video $\left(\mathrm{MP}_{3}, \mathrm{MP}_{4}\right)$, las cámaras y videocámaras digitales, los discos compactos, las consolas de juego y memorias USB y discos duros portátiles, entre otros tantos.

En lo que atañe al software, se encuentran los exploradores o navegadores de Internet (Firefox, Chrome, Internet Explorer, Safari), los sistemas operativos para computadores con todos sus aplicativos y programas (Linux, Mac, Windows), los sistemas operativos para teléfonos celulares (Android, iOS) y todo tipo de aplicativos como el popular WhatsApp, Skype o Tango.

Entre los servicios que ofrecen las TIC se pueden citar el correo electrónico, los motores de búsqueda de información (research engines) tanto buscadores como metabuscadores (Google, Yahoo, Bing, Altavista, AlltheWeb, etc.); las web pages que permiten servicios en línea tales como la banca online, el comercio electrónico, el gobierno electrónico (pago de impuestos, servicios públicos, realización de trámites y descarga de certificados, entre otros) y toda una suerte de servicios como las páginas de Amazon o eBay; las empresas proveedoras de Internet o ISP por sus siglas en inglés (Internet service provider) entre las que podemos mencionar a Claro, Telefónica o ETB (para el caso colombiano), el e-learning (educación virtual), los videojuegos, los blogs y servicios peer to peer (P2P), el almacenamiento y recuperación de datos en la nube (e-cloud), entre otros.

El presente trabajo se enfocará en el estudio de Internet y su relación con el derecho, aunque si bien la mayoría de las TIC transmiten información utilizando de alguna manera la plataforma de la gran red de computadores y servidores que conocemos con el nombre de Internet ${ }^{3}$.

El problema de investigación del proyecto de investigación al que contribuye este artículo es

\footnotetext{
3 La expresión Internet proviene de las palabras interconnected networks (redes interconectadas), por ello lo más adecuado sería referirse a "la Internet", como una red que es.
} 
el siguiente: ¿cómo se está resolviendo el aparente conflicto entre los derechos a la intimidad, a la libre expresión y los requerimientos de la seguridad en casos generados en Internet? Se advierte que este documento es la parte introductoria de un estudio más amplio, por lo que aquí no se desarrollan los contenidos que se asocian con los mencionados derechos a la intimidad, la libre expresión y el tema de la seguridad; por ello, el objetivo del artículo es presentar un material para los abogados y juristas que deseen iniciarse en los estudios sobre Internet y derecho o de forma más amplia, sobre las TIC y el derecho.

En este sentido, se exponen los aspectos más destacados del entorno tecnológico que permiten la comunicación por este medio, así como sus características y temáticas más relevantes para la disciplina jurídica. Se emplea un método cualitativo $e$ inductivo, usando fuentes primarias $y$ secundarias y técnicas de revisión documental, estudios de casos y observación directa.

El documento se divide en tres partes además de la introducción. En la primera se abordan aspectos fundamentales para aproximar a los abogados al conocimiento de Internet y su ambiente tecnológico; para ello se presenta el origen, evolución, componentes y administración de Internet. En la segunda se hace un intento por unir el Internet con las preocupaciones jurídicas para de este modo, poder hablar del "derecho de Internet", su definición, características y consecuencias jurídicas, ilustradas con algunos ejemplos de casos colombianos; seguidamente se revisan las temáticas más recurrentes en las disputas generadas en la red como medio de comunicación generalizado y finalmente, se hace una breve reseña sobre la evolución normativa y jurisprudencial en Colombia. En la tercera se dan las conclusiones.

\section{A. Aspectos básicos sobre Internet}

\section{Origen y desarrollo de Internet, la www y el correo electrónico}

La Internet aparece en la década de los sesenta en Estados Unidos. Se atribuye a J.C.R. Lic- klider, jefe del programa de investigación en Advanced Research Projects Agency en agosto de 1962, la idea de crear una "red galáctica" de computadoras conectadas recíprocamente. En 1965 los ingenieros L. Roberts y T. Merrill conectaron mediante una línea telefónica, el computador Q-32 ubicado en California, con el computador TX-2 situado en Massachusetts (Consejo de Estado, 2001) .

En 1969 se crea la red ARPANET inicialmente buscando objetivos militares y con protocolos de transferencia problemáticos y lentos, pero va apareciendo la conmutación de paquetes o grupos de información que tienen dos elementos: (i) la información enviada y (ii) la información de control, que indica la ruta a seguir hasta el destino. Los datos son divididos en listas de ceros y unos, lo cual origina el esquema binario de los bits que potenciará la transmisión de datos ${ }^{5}$.

Paralelamente se dio origen al correo electrónico, en la actualidad uno de los servicios más populares de Internet; para algunos como Peter (s. f.), el correo electrónico se desarrolló antes que ARPANET, pues fue en 1965 cuando se usó el primer sistema de correo electrónico llamado mailbox en el Massachusetts Institute of Technology. Antes que Internet se desarrollara, los correos electrónicos solo podían ser enviados por usuarios de un mismo computador (usualmente alrededor de cien personas), conectados desde sus escritorios mediante dumb terminals (terminales mudos), los cuales no tenían capacidad de almacenamiento ni memoria.

Esta decisión de la Sala de Consulta y Servicio Civil del Alto Tribunal de lo Contencioso Administrativo es un importante precedente sobre Internet, los dominios y el carácter público del dominio ".co".

5 Bit es el acrónimo de binary digit; el bit es el dígito binario que puede representar el valor de 0 o 1 y se ha convertido en la unidad básica de la informática. Ocho bits forman un byte, y en el sistema binario 1024 bytes constituyen el kilobyte, 1024 kilobytes forman un megabyte, 1024 megabytes son un gigabyte, 1024 gigabytes componen un terabyte, luego siguen el petabyte, el exabyte, etc. Sin embargo, en el sistema decimal las cosas cambian: 1000 bytes son un kilobyte, 1000 kilobytes son un megabyte y así sucesivamente. 
La evolución de Internet permitió la comunicación entre computadores, pero la transmisión del correo se volvió más compleja, pues se necesitaba enviarlo a una dirección determinada y colocarlo en un buzón. Por ello, en 1971 Ray Tomlinson, introdujo el símbolo arroba “@” del teclado para separar al usuario del servidor donde se ubica el buzón de correo (name-of-the-user@name-of-the-computer).El símbolo@ en inglés significa at y en español "en"; entonces, una dirección como gjimen00@ gmail.com, significa el usuario (gjimen00) "en" Gmail, esto es, el servidor donde los mensajes permanecen temporalmente mientras se envían al destinatario o son consultados (Tecnocosas, s. f.). El problema estaba resuelto.

Continuando con la evolución de Internet, hacia 1973 aparecen los protocolos TCP (transmission control protocol) que facilitan enormemente el flujo de información al garantizar la entrega de datos en su destino o puerto. Otro gran avance en Internet fue la creación de un protocolo propio o IP (Internet protocol) que básicamente sirve para ubicar la dirección a la cual se va a enviar la información; estos IP están compuestos de una serie de números en cuatro grupos con dos o tres dígitos cada uno (en realidad son 32 números bit), lo que dificulta su identificación y memorización rápida por parte de los usuarios (por ejemplo, 123.49.12.412); de esta manera surgió la necesidad de identificar las direcciones IP, y así emergieron los dominios de Internet, que indican la dirección de un sitio en el ciberespacio, utilizando nombres en lugar de números. Todo nodo de Internet, ya sean servidores, routers o PC, tiene asignado un IP y por ello su dirección puede ser reconocida dentro del sistema (ver más adelante: dominios y URL).

Hoy por hoy, Internet ofrece variados servicios tales como: correo electrónico, chats, bases de datos, www (world wide web) y transmisión de imágenes, música y video. Para algunos, los más importantes servicios de Internet son el correo electrónico y la www, debido a que son los más populares (Svantesson, 2007).
El correo electrónico, como ya se indicó, fue una de las primeras aplicaciones de Internet y hoy admite no solo enviar mensajes sino adjuntar todo tipo de archivos con sonido e imágenes. En la actualidad es normal que una persona del común posea varias cuentas personales de correo electrónico, debido a la gratuidad y utilidad de las mismas, también es muy probable que tenga un correo electrónico institucional, esto es, aquel ofrecido por la empresa donde trabaje. Si bien el correo electrónico se ha popularizado, su uso puede ir en declive debido a la aparición de otras formas más ágiles de envío de información, tales como WhatsApp o Skype.

La www (también conocida como W3 o "la web") ha tenido un gran éxito hasta el punto que muchos la confunden con Internet; está compuesta por un conjunto de protocolos que permiten al usuario, de forma sencilla, la búsqueda de todo tipo de archivos de hipertexto (Guerra, 2012). La inventó R. Carillau y T. Berners-Lee en el Laboratorio Europeo de Física de Partículas Elementales en Suiza a comienzos de los años noventa y su rasgo principal es que todos los recursos y aplicaciones de la www usan formato HTTP (hypertext transfer protocol) (Svantesson, 2007).

\section{Administración de Internet}

Debido al crecimiento de la red, se crearon dominios genéricos o de primer nivel, sobre los cuales empezaron a registrarse los nombres. Los dominios se componen de una raíz y una extensión; la primera identifica al usuario y la segunda al ámbito de actividad o el país en el cual se registra la raíz; por ejemplo, en la dirección www. tumoto.com, la raíz es "tumoto" y la extensión es ".com", que en este caso es de primer nivel o TLD (top level domain), pues puede haber extensiones de segundo nivel de dominio como sería el caso de www.tumoto.com.co, en el cual ".co" sería dominio de segundo nivel (aunque pueda ser de primer nivel, en el caso que siga inmediatamente a la raíz). Así las cosas, existen dominios genéricos o de cobertura global; los más frecuentes usan las siguientes extensiones: 
.com (comercial o de negocios), .org (organizaciones, por lo corriente sin ánimo de lucro), .net (comunicaciones), .edu (instituciones educativas como las universidades), .gov (gubernamental u oficial), .int (organizaciones internacionales), .mil (organizaciones militares), .jobs (organizaciones del trabajo) y .travel (organizaciones de viajes).

De igual modo, los dominios de país utilizan dos letras para identificarlos; estas terminaciones se tomaron de la norma ISO 3166 de 1974 sobre orígenes y destinos del transporte internacional (Consejo de Estado, 2001). Entre ellos tenemos, por ejemplo: .co (Colombia), .nl (Países Bajos), .ar (Argentina), .mx (México), .es (España), etc. Los dominios son importantes porque indican la dirección o lugar tanto de origen como de destino de la información de las páginas de Internet; esto en materia de establecer la jurisdicción o competencia de los jueces y autoridades puede ser crucial, como se verá más adelante.

Un concepto relevante es la URL (uniform resource locator) o localizador uniforme de recursos, que es la dirección global de documentos y otro tipo de recursos en la W3, en otras palabras, es el modo estándar de identificar una dirección de un sitio en Internet. La primera parte de la URL identifica el protocolo usado para obtener el recurso solicitado y puede ser http:// para páginas web oftp:// para descarga de archivos; la segunda parte de la URL señala el dominio de primer nivel, de segundo nivel y la ruta completa si la información se encuentra en un subdirectorio, según el caso. Ejemplos de URL son: http://www.internetjurisdiction.net/ observatory/, o ftp://fpt.descargas.com.

Paralelo al crecimiento de Internet y la asignación de los dominios y direcciones, aparecieron organismos encargados de su administración. En principio fue la Internet Assigned Numbers Authority con una alta intervención del gobierno de Estados Unidos. En 1998 se transformó en la ICANN (Internet Corporation for Assigned Names and Numbers), una organización sin fines de lucro y de utilidad pública que coordina de manera global los identificadores de Internet para su funcionamiento estable y seguro; su sede está en California en el Instituto de Ciencias de la Información de la Universidad del Sur de California.

ICANN puede delegar en cada país o región la administración de los dominios, como sucedió inicialmente en Colombia cuando la Universidad de los Andes tenía tal servicio antes que el Estado colombiano tomara cartas en el asunto mediante el concepto del Consejo de Estado en 2001 que permitió la intervención del Ministerio de las Comunicaciones de ese entonces (Consejo de Estado, 2001). ICANN está administrada por una junta directiva de catorce miembros (seis representantes de secciones específicas y ocho representantes de interés general para dar cabida a las diferentes circunscripciones regionales en el ámbito mundial), y un director ejecutivo que nombra la junta.

Otro aspecto importante para entender Internet es el sistema de acceso a la red, en el que puede desempeñar un papel significativo el tema del control sobre la circulación de información. Por lo general la disposición del servicio concibe un sistema de cuatro niveles o escalones (four-tier system); el usuario normal o persona común y corriente que desde su casa u oficina accede a Internet está en el cuarto nivel; el proveedor del servicio de Internet (ISP) es el tercer escalón, pues facilita al usuario el acceso a la red (por ejemplo, Claro, ETB o Movistar); la segunda grada es el proveedor de acceso a Internet (Internet access provider, IAP) que son los dueños físicos de la red, cuentan con el equipamiento y las líneas de telecomunicaciones para el acceso, por ello son compañías que alquilan sus servicios de acceso a los proveedores de servicios y en ciertos casos actúan como ISP (por ejemplo, AT\&T WorldNet, IBM Global Network, MCI, Netcom, UUNet y PSINet). Finalmente, en la primera grada está el portal del gobierno que permite a los IAP conectarse y de esta forma acceder a la red global. Algunos países contemplan en su legislación, la aplicación estricta del sistema controlado por el Estado (por ejemplo China o Cuba), pero la mayoría no puede ejercer un control de frontera 
efectivo debido a que el flujo de comunicación por Internet pasa a través de una multitud de proveedores públicos y privados, sin mayores restricciones legales (Svantesson, 2007).

En todo caso, el asunto del manejo y administración de Internet desborda los intereses meramente corporativos, implicando los intereses de todas las poblaciones y Estados. Hoy existen otros organismos y foros como la Unión Internacional de Telecomunicaciones y el Internet Governance Forum, este último con participación multisectorial sobre los problemas de Internet en el mundo globalizado.

En el tema específico del derecho, vale mencionar al proyecto europeo Internet \& Jurisdiction Project, preocupado por conciliar la naturaleza transfronteriza de Internet con los sistemas judiciales de carácter nacional; este se financia de donaciones de organismos públicos, privados y ONG. El observatorio de Internet \& Jurisdiction Project creó una colección de casos judiciales desde 2012 alrededor del mundo (aproximadamente relata veinte casos cada mes), que relaciona tópicos como la adopción de nueva legislación, proyectos de ley, bloqueo de contenidos ofensivos, multas por violación de leyes locales, ley aplicable y jurisdicción, y casos judiciales, entre otros ${ }^{6}$.

\section{B. Derecho e Internet: cuestiones fundamentales}

Como se indicó, el aumento de todo tipo de transacciones en Internet ha generado polémicas en cuanto a su regulación y control gubernamental, así como no pocos conflictos jurídicos en los estrados judiciales. De esta manera, ha aparecido un nuevo campo en los estudios y práctica jurídica denominado derecho de Internet, derecho del ciberespacio, derecho informático o derecho de las tecnologías de la información; sin embargo, autores reconocidos prefieren referirse a "derecho de Internet" (Internet law) antes que a derecho del ciberespacio (cyberspace law) o

6 Ver por ejemplo, https://www.internetjurisdiction.net/ derecho de las tecnologías de la información (information technology law), debido a su especificidad y definido ámbito de aplicación (Svantesson, 2007). Incluso, el profesor Arno Lodder de la Universidad Libre de Ámsterdam, estableció los "Diez Mandamientos" del derecho de Internet, para los abogados que se ocupan de estos asuntos (Lodder, 2013).

Si bien en español ha sido acogido con alguna fortuna el término "derecho informático", entendido como "el conjunto de principios y normas que regulan los efectos jurídicos nacidos de la interrelación de sujetos en el ámbito de la informática y sus derivaciones, especialmente en el área denominada 'tecnología de la información" (Tato, s. f., p. 1), dicho concepto, a nuestro entender desborda el específico campo de la Internet para ocuparse en general, de la informática y las TIC y por otro lado, parece centrar su atención en los aspectos de la regulación normativa, descuidando otros temas del derecho como la judicialización y resolución de conflictos en los ambientes virtuales, aunque de forma indirecta, la cuestión de la regulación conduce a la de la jurisdicción e imposición de medidas.

Podemos definir el derecho de Internet como aquel campo del derecho que focaliza su atención en la regulación, jurisdicción, juzgamiento, control e imposición de medidas tendientes a garantizar los derechos y cumplir las obligaciones originadas en las transacciones, usos e intercambios de información que utilizan como medio la red global interconectada de computadoras. Se trata de un campo en donde interactúan tanto el conocimiento del derecho como la lógica de la tecnología de Internet y que necesariamente implica las tradicionales ramas de derecho como el civil, el penal, el laboral, el administrativo, el comercial, el internacional y el constitucional. De este modo, las aproximaciones sobre derecho e Internet pueden resumirse en los siguientes dos puntos:

First, the classic legal approach is to start with the legal norm, and see whether internet phenomena are covered by existing norms 
[...] Second, the internet can be taken as starting point, so to think about possible norms without considering the existing normative framework. This approach can lead to new, refreshing insights (Lodder, 2013, p. 264).

Para entender de manera propedéutica este nuevo campo, es necesario primero determinar las características de Internet para luego pasar a los tópicos o temáticas más relevantes para el derecho, que se presentan dentro de la red.

\section{Características de la comunicación por Internet y consecuencias}

Estas características, relacionadas con el derecho, se tornan en dificultades ya sea para fijar la ley aplicable a un caso, el establecimiento de la jurisdicción o juez de conocimiento, la coalición de jurisdicciones nacionales y de soberanía, problemas para el cumplimiento de una orden o sentencia, la instauración de la responsabilidad y la valoración del material probatorio, entre otras tantas. Ahora bien, las características de Internet varían según sus diferentes servicios. Por ejemplo, la cualidad one to everyone aplica perfectamente para la www, pero de manera restringida para el correo electrónico, el chat y ciertas redes sociales, en las que la comunicación sigue los estándares de la comunicación one to one; con lo cual, hay que tener cuidado a la hora de determinar las consecuencias. Entre las más importantes características de Internet, vale la pena mencionar las siguientes:

\section{Carácter transfronterizo (crossborder o borderless)}

Significa que las comunicaciones y la información que circula por Internet penetran fácilmente las fronteras territoriales. Lo anterior quiere decir que la información fluye libremente entre los países sin que haya controles de aduana como acontece con otras formas de comunicación. Este es el rasgo general, no obstante, ciertos países pueden imponer algún tipo de restricciones fronterizas a la libre circulación de la información dentro de su espacio aéreo o espectro electromagnético ${ }^{7}$. Se trata de países en los cuales el papel del Estado es preponderante y por ello, los derechos a la libertad de información y de opinión están limitados, en palabras de Maquiavelo, por "razones de Estado" (1993, p. 73).

Esta cualidad se refiere al amplio espectro de cubrimiento de la red, lo que la hace transfronteriza o transjurisdiccional; baste por ahora citar un ejemplo ilustrativo en Colombia:

Con ocasión de una acción de tutela en 2004, se solicitó al juez el amparo del derecho a la intimidad por una persona que estaba recibiendo mensajes con publicidad no deseada en su correo electrónico, pues pese a las solicitudes reiteradas, el emisor de los mensajes continuaba enviando esta información. El tema controversial fue que la acción de tutela se presentó ante un juez que en principio no tenía competencia puesto que su despacho estaba ubicado en el municipio de Rovira (Tolima), mientras que tanto el emisor como el receptor de los mensajes tenían domicilio en Bogotá y era allí donde el juez tenía jurisdicción.

No obstante, el juez de Rovira consideró que debido al carácter amplio de Internet, los mensajes podrían ser enviados y leídos en cualquier parte del país, de suerte que el correo electrónico del tutelante sería su domicilio virtual y de este modo, esto le otorgaba jurisdicción para conocer y decidir el caso, como en efecto ocurrió ${ }^{\text {. }}$

\section{Independencia geográfica}

Estrechamente relacionada con la anterior característica, implica al menos tres cosas: (i)

7 El espectro electromagnético se refiere al campo donde se mueven todas las longitudes de onda de las radiaciones electromagnéticas posibles y que permiten las comunicaciones; en Colombia, dicho espectro electromagnético es parte integral del territorio según el artículo 101 de la Constitución Política.

8 Una crítica a esta decisión se encuentra en Manrique (2011). 
para quien ofrece información, significa la facilidad para que la comunicación por Internet esté disponible a mucha gente a través de las fronteras; (ii) para quienes buscan información, la ubicación geográfica de la información es virtualmente irrelevante: "it could be said to be possible to effortlessly move over great geographical distances, and between different states" (Svantesson, 2007, p. 32); y (iii) el costo de la comunicación es relativamente independiente de la distancia, en cambio el servicio postal o el teléfono incrementan con la distancia.

Un ejemplo lo constituye la reglamentación establecida en la ley 1564/2012 o Código General del Proceso (CGP) sobre la prueba de normas jurídicas. Ha sido tradición que las normas de carácter nacional no requieran probarse y por ello, no es menester incorporar copia de las mismas al proceso; pero las normas no nacionales y las leyes extranjeras deben aducir copia al proceso. Este último requisito no será necesario si la norma se encuentra publicada en la página web de la entidad respectiva, así señala el CGP:

Artículo 177. Prueba de las normas jurídicas. El texto de normas jurídicas que no tenga alcance nacional y el de las leyes extranjeras, se aducirá en copia al proceso, de oficio o a solicitud de parte.

La copia total o parcial de la ley extranjera deberá expedirse por la autoridad competente del respectivo país, por el cónsul de ese país en Colombia o solicitarse al cónsul colombiano en ese país.

También podrá adjuntarse dictamen pericial rendido por persona o institución experta en razón de su conocimiento o experiencia en cuanto a la ley de un país o territorio fuera de Colombia, con independencia de si está habilitado para actuar como abogado allí.

Cuando se trate de ley extranjera no escrita, podrá probarse con el testimonio de dos o más abogados del país de origen o mediante dictamen pericial en los términos del inciso precedente.

Estas reglas se aplicarán a las resoluciones, circulares y conceptos de las autoridades administrativas. Sin embargo, no será necesaria su presentación cuando estén publicadas en la página web de la entidad pública correspondiente (énfasis fuera del original).

Esta excepción se basa en el conocimiento público de amplio espectro que generan las páginas de Internet de los municipios, distritos y departamentos.

\section{La comunicación es virtualmente ins- tantánea}

Diferente a otras formas de comunicación en las cuales a mayor distancia mayor tiempo, aquí la diferencia temporal entre consultar una URL ubicada en la misma ciudad y consultar otra localizada al otro lado del mundo, es casi irrelevante.

\section{Amplio o popular uso}

Internet invadió nuestras vidas. Además de las características mencionadas, el éxito de este tipo de comunicación descansa en los siguientes aspectos adicionales: (i) bajo nivel de requerimientos técnicos y económicos al usuario; es decir, que es accesible a una persona promedio, sin un nivel de educación específico o altas barreras económicas; y (ii) limitada dependencia del idioma, Internet ha facilitado la comunicación entre diferentes idiomas haciendo posible la comunicación global, gracias a la disposición de herramientas de traducción en línea de manera fácil, rápida y oportuna.

\section{Portabilidad}

Aunque esta característica es compartida por otras formas de comunicación como el correo postal o el teléfono, en el caso de Internet es más extrema. La portabilidad es un término de la 
informática con dos significados: (i) la capacidad de un programa o sistema para ejecutarse en diferentes plataformas o arquitecturas, con mínimas modificaciones, y (ii) la facilidad con que se puede transportar un dispositivo electrónico como una computadora o una consola (Alegsa, s. f.); de aquí el término computador portátil. La portabilidad numérica, por su parte, hace referencia a la posibilidad de trasladar el mismo número telefónico de una compañía a otra.

En la comunicación de Internet, significa la cantidad de páginas web, servidores, computadores y dispositivos disponibles en cualquier lugar, que pueden así mismo, acceder a la misma página o información de forma simultánea e independiente: "Related to this point is that although there is always one particular place where people access the internet, this place can be anywhere" (Jiménez y Lodder, 2015, p. 268). Esto hace posible que una misma página pueda ser consultada por distintos servidores ubicados en diferentes partes del planeta o que una página de Internet tenga sus textos almacenados en un servidor ubicado en Bogotá, mientras que sus fotografías están en un servidor situado en Ciudad de México.

Además de lo precedente, no es necesario llevar o portar el propio computador para ingresar a la red: podemos acceder a Internet desde cualquier terminal, sin importar el lugar del mundo, lo importante es que esté conectado. Cuando accedemos a nuestra cuenta de correo electrónico, por ejemplo, desde diferentes equipos y desde diferentes ciudades, estamos ante la presencia de la portabilidad, pues solo precisamos de introducir el nombre del usuario y la clave para consultar o enviar mensajes. Entonces, para el usuario de Internet, la ubicación geográfica del servidor que le lleva la información, es intrascendente.

La portabilidad es una de las particularidades más relevantes en cuanto a determinar el lugar de los hechos o la ubicación de las partes dentro de un contrato o pleito judicial, como lo ilustra el siguiente caso.
En el año 2010, cinco personas mediante una transacción en línea fraudulenta originada en Barranquilla, sustrajeron dinero de la cuenta bancaria del Centro Comercial Campanario en Popayán (Jiménez, 2015). La denuncia se presentó ante el Tribunal Superior de Barranquilla por ser el competente de acuerdo con el lugar donde los actos ilícitos se cometieron; la conducta se tipificó como "hurto por medios informáticos y semejantes". Pese a ello, el tribunal no asumió la competencia y envió el proceso al Tribunal de Distrito de Popayán, donde el titular de la cuenta bancaria tenía su domicilio. Este tribunal, a su vez, remitió el caso a la Corte Suprema de Justicia para que determinara la competencia. En este caso, la Corte Suprema de Justicia (2010b), sostuvo lo siguiente:

[...] la afectación del bien jurídico del patrimonio económico particular, ciertamente se produjo en la ciudad de Popayán, toda vez que fue allí donde se realizó el despojo del dinero objeto del apoderamiento ilícito, sin importar desde dónde se haya originado la maniobra que transfirió el dinero y cuál su destino final (énfasis fuera del original).

Debido a la portabilidad de Internet, el acento se pone en el lugar donde el daño o lesión tuvo lugar, no donde los comportamientos ilícitos se realizaron y esto genera un cambio de apreciación para establecer el juez competente.

\section{Difícil control}

Significa que debido a la naturaleza descentralizada de Internet, su control central se hace muy difícil; pese a ello, existen ciertos niveles de control como el que realiza el gobierno sobre los IAP dentro de un país respectivo, o el que ejecutan los ISP sobre sus suscriptores. El punto aquí es la carencia de un control centralizado mundial, lo que limita la capacidad de hacer cumplir ciertas decisiones y reglamentos. Como ejemplo, de las dificultades para el control, se presenta el siguiente caso a propósito de la protección de datos personales en las redes sociales. 
En noviembre de 2014, la Superintendencia de Industria y Comercio de Colombia decidió que no tenía jurisdicción sobre el manejo de la información personal almacenada en Facebook, porque dicha compañía no tenía su domicilio en Colombia. No obstante, frente a las críticas a esta postura por desconocer los alcances de la ley 1581/2012 sobre el Sistema de Protección de Datos Personales (Remolina, 2015), y al análisis posterior que efectuó la propia Superintendencia, en marzo de 2016 cambió su posición al respecto, al establecer que sí tiene facultad para garantizar el tratamiento de datos personales de los colombianos que compartan información personal en las llamadas redes sociales de Internet; se rescata lo siguiente:

De todo lo hasta aquí anotado, es dable concluir que:

(i) en las plataformas de comunicación en línea, tales como Facebook, Instragram o Linkedln, efectivamente se realiza tratamiento de datos personales, verbigracia, contactos, fotografías, o vídeos, mediante la creación de un perfil personalizado, alojamiento, conservación, publicación, circulación y supresión de información personal, la utilización de los datos con fines de comercialización, la localización de información de una persona y el acceso al perfil público o privado.

(ii) el proveedor de servicios de redes sociales es responsable del tratamiento de datos en las plataformas de comunicación en línea, en lo que respecta al derecho de Hábeas Data.

[...] Por lo anterior, repara esta Entidad en que no puede ser otra la interpretación que del mismo artículo 2 de la mencionada ley se realice, dado que, tal y como lo expuso la Corte Constitucional al analizar la constitucionalidad del ámbito de aplicación de la ley estatutaria de protección de datos personales, nos encontramos "[e]n un mundo globalizado en el que el flujo transfronterizo de datos es constante, la aplicación extraterritorial de los estándares de protección es indispensable para garantizar la protección adecuada de los datos personales de los residentes en Colombia, pues muchos de los tratamientos, en virtud de las nuevas tecnologías, ocurren precisamente fuera de las fronteras. Por tanto, para la Sala se trata de una medida imperiosa para garantizar el derecho al habeas data (Superintendencia de Industria y Comercio, 2016; énfasis fuera del original).

\section{Convergencia y conectividad con otras formas de redes}

La estructura o ingeniería de Internet permite que otros medios de comunicación se ofrezcan a través de su red como la televisión, la radio, la prensa o la telefonía. Esto se debe al sistema de protocolos que rigen Internet, los cuales son abiertos y flexibles por lo que cualquier forma de red puede ser conectada y compartir sus datos e información. Este rasgo es el que menos ofrece problemas con asuntos de orden jurídico, en principio (Svantesson, 2007).

El caso de un periódico que aparece en versión en línea, es un ejemplo ilustrativo. Se trata de una acción de tutela accionada por una persona que apareció nombrada en una noticia titulada "Empresa de trata de blancas" publicada el 29 de agosto de 2000 en la página web de la Casa Editorial El Tiempo. Según la accionante, fue vinculada a una investigación penal en ese momento, pero luego, en 2008 se profirió la prescripción de la acción penal en su favor; posteriormente, en diciembre de 2013 solicitó a la casa editorial que eliminara la noticia sin obtener respuesta favorable. De este modo, interpuso acción de tutela para que se le ampararan los derechos al buen nombre, la intimidad, a la petición, el debido proceso y el derecho al trabajo; solicitó que la Casa Editorial El Tiempo bajara y borrara los contenidos de la noticia de todos los buscadores, en especial de Google, cuando se utilizara su nombre como descriptor de búsqueda. La Corte Constitucional (2015) al analizar el caso en revisión, declaró: 
Adicionalmente, ha de considerarse una circunstancia que particulariza la situación de la accionante, consistente en que la información suministrada por el medio de comunicación, que resultaría lesiva de sus derechos fundamentales, se encuentra disponible de forma permanente al estar publicada en internet. La constante accesibilidad de la noticia hace que el deber de actualización a cargo de su autor se vuelva particularmente sensible, pues haber sido objeto de una publicación noticiosa cuya disponibilidad para terceros ha decaído con el paso del tiempo no entraña las mismas consecuencias desde una perspectiva de derechos fundamentales que el estar sujeto de forma ininterrumpida al escrutinio público debido a que dicha información puede ser conocida por todos en cualquier momento, pese a que no aparece completa porque informa parte de los hechos pero no su desenlace (énfasis fuera del original).

Como resultado, la Corte ordenó a la Casa Editorial El Tiempo: (i) actualizar la información publicada en su página web, para que se informara que dicha persona no fue vencida en juicio; y (ii) neutralizar por medio de la herramienta técnica robots.txt, metatags $\mathrm{u}$ otra similar, la posibilidad de libre acceso a la noticia "Empresa de trata de blancas" con la simple digitación del nombre de la tutelada en los buscadores de Internet.

\section{Infinito número de destinatarios}

Diferente a la comunicación one to one del teléfono, el fax o aún del correo electrónico, este es one to many; es decir, el envío de la información puede llegar a múltiples y desconocidos destinatarios. Ahora bien, esta peculiaridad es compartida por otras formas de comunicación como la televisión o la radio, pero la combinación de esta cualidad con otras ya mencionadas como la portabilidad e independencia geográfica, hace más difícil su control como se expresó arriba (Svantesson, 2007).

\section{Anonimato}

La comunicación por Internet facilita el intercambio de información entre personas cuyos detalles particulares o individualización es difícil de establecer; además, muchos usuarios utilizan alias o nombres no reales, ocultan su información básica o simplemente suplantan a otros; por eso muchas veces es casi imposible determinar el nexo de una persona en particular con la información que ha sido alojada en Internet. Para efectos judiciales esto es crucial pues: "If you want to link a particular action to jurisdiction you need to know who is behind it" (Jiménez y Lodder, 2015, p. 269).

El caso de Jerónimo A. Uribe en 2010, hijo del entonces presidente de la República, ilustra las características crossborder, de independencia geográfica y de anonimato de Internet. En el caso, se presentó una denuncia por la configuración del tipo penal "instigación a delinquir agravado", debido a que en Facebook apareció un grupo llamado: "Me comprometo a matar a Jerónimo Alberto Uribe, hijo de Álvaro Uribe". La policía encontró y capturó a una persona que presuntamente había creado el grupo, desde el municipio de Chía. La imputación y acusación fueron puestas por el fiscal en el Tribunal Superior de Bogotá, por lo que la defensa cuestionó la jurisdicción de dicho tribunal y sostuvo que como la conducta fue originada en Chía correspondía su conocimiento al Tribunal de Cundinamarca, donde se encuentra Chía (Jiménez, 2015). El asunto se elevó ante la Corte Suprema de Justicia (2010a) para definir el conflicto de competencia y el alto tribunal dispuso que debido a que Facebook tiene:

[...] cobertura mundial y transnacional, no permite precisar que haya tenido ocurrencia en el municipio de Chía... Frente a tales supuestos, por consiguiente, en que el factor territorial no presta utilidad para establecer la competencia, es necesario acudir a las pautas del inciso segundo del artículo 43 del estatuto procesal penal. 
La Corte señaló que en estos casos, de acuerdo con el Código de Procedimiento Penal, el juez de conocimiento es aquel donde la Fiscalía coloca la denuncia, y esto fue en Bogotá, por lo que ese tribunal tiene la jurisdicción.

La decisión final fue declarar al acusado inocente por falta de pruebas que le llevaran certeza al juez acerca de que dicha persona había creado el grupo en Facebook, esto en gran medida, por el anonimato que caracteriza las comunicaciones por Internet.

\section{Fácil reproducción, copia y reenvío de información}

Esta característica se refiere a la posibilidad casi infinita que tiene un usuario de bajar información, copiarla en una memoria o computador y reenviarla o redistribuirla a múltiples destinatarios, quienes a su vez pueden hacer lo mismo. A esto se debe añadir, que en dicho proceso no hay pérdida de la calidad de dicha información, como sucede en otros medios no digitales. Para los asuntos jurídicos es crucial, pues en temas relacionados con violaciones a la intimidad o difamación se hace complicado establecer el origen y aplicar las respectivas medidas correctivas: "Even if the original source can be traced, there can be many others already linking to or hosting the same information. And, again, they can be rooted in a number of jurisdictions" (Jiménez y Lodder, 2015, p. 268).

\section{Temas cruciales sobre derecho e Internet}

Los primeros estudios académicos sobre Internet y derecho (Quinto, 2003), identificaron una serie de temáticas que originaban controversias legales en Internet:

1. Propiedad intelectual y comercio electrónico.

2. Derecho a la privacidad.

3. Libertad de expresión.

4. Espacios virtuales, derechos públicos y privados.

5. Regulación de relaciones exteriores de la Internet.
6) Jurisdicción y comunidad mundial.

7) Proveedores de servicios de Internet (IPS, por sus siglas en inglés).

A los siete anteriores, habría que agregar sin duda, el tema de los delitos informáticos. Para Svantesson (2007) las áreas del derecho internacional privado más susceptibles son: (i) los contratos comerciales; (ii) los contratos del consumidor; (iii) el daño por difamación y (iv) la relación entre contratos y perjuicios. Por su parte, un estudio analizó 41 casos judiciales clave en Estados Unidos; del análisis de estos se concluyó que el asunto de la propiedad intelectual era el más frecuente en controversias de Internet (62\%), seguido por las controversias sobre difamación y agravios (Reidenberg, 2013).

Por otro lado, se podrían agrupar los diferentes temas, según las especialidades del derecho, para obtener el siguiente resultado: (i) propiedad intelectual, marcas, comercio electrónico y contratos (derecho civil y comercial, derecho internacional privado, según el caso); (ii) delito informático y difamación (derecho penal); (iii) contratos, relaciones laborales y seguridad social (derecho laboral); (iv) derecho a la privacidad y a la libertad de expresión (derecho constitucional); (v) regulación de servicios de Internet y gobierno electrónico (derecho administrativo); (vi) gobierno de Internet (derecho internacional); y (vii) jurisdicción en Internet (comprende todas las especialidades).

Investigaciones recientes siguen centrándose en la cuestión de cómo se establece la jurisdicción en Internet. En las disputas jurídicas sobre Internet el concepto "jurisdicción" es mucho más amplio que el concepto del derecho nacional, concretado en la capacidad del juez para conocer y decidir un caso ${ }^{9}$, debido a que las controversias muchas veces implican el derecho internacional (tanto público como privado), el vocablo jurisdicción

9 En Colombia se utiliza la expresión "competencia" con significado similar, pero dicho término no es preciso y además es polisémico, pues se refiere también a las aptitudes, conocimientos y experticia que se requieren para desempeñar un determinado trabajo. 
puede entenderse o usarse según los siguientes significados:

- La capacidad regulatoria del Estado o autoridad; es decir, la facultad para prescribir y regular ciertas conductas, ya mediante la ley u otro tipo de norma jurídica; esto comporta la facultad de imponer sanciones y ejecutarlas.

- El territorio de un Estado, o el área de un determinado distrito o foro. Aquí el territorio es el elemento básico, ya sea en el tema del derecho internacional o en el derecho interno para fijar la división en distritos y circuitos judiciales.

- La capacidad de una corte, tribunal o juez para conocer y decidir una disputa judicial (aquí se recoge el origen etimológico: decir el derecho: iuris-dictio).

De igual manera, es importante señalar que el derecho internacional privado consta de tres temas muy relacionados entre sí: (i) la jurisdicción, ya enunciada; (ii) la elección de la ley-determinar qué ley debe aplicarse a un caso en concreto-; y (iii) el reconocimiento y ejecución, que se refiere al efecto directo y obligación de cumplimiento de una sentencia extranjera (Wang, 2010).

Según Kohl (2010) el principal problema del derecho de Internet es que las normas jurídicas fueron creadas según el supuesto de que las actividades estaban localizadas geográficamente y en consecuencia, la ubicación se convirtió en el criterio preponderante para conocer la jurisdicción y competencia tanto de los jueces como de las autoridades regulatorias en general: "Finally, States are today struggling with accommodating these difficult events within their allocation rules based on location, so much so that there have been some calls to abandon the territorially based system of regulation" (Kohl, 2010, p. 3).

En un principio, los juristas del derecho reaccionaron al asunto de la regulación jurídica de Internet de dos maneras diferentes (Jiménez,
2015): (i) la creencia de que las normas jurídicas tradicionales se podrían aplicar a las transacciones virtuales, sin problema alguno: "Se supuso, en un primer momento, que la transposición pura y simple de las reglas del mundo 'real' a lo virtual sería suficiente para evitar anomia jurídica" (De Lucca, 2012, p. 11); y (ii) la postura que señalaba la necesidad de instaurar nuevas reglas jurídicas provenientes de la actividad del ciberespacio: "Their central assertion was that the traditional jurisdictional rules based on geographic location are not transferable to the transnational Internet" (Kohl, 2010, p. 11).

Sobre el tema de la jurisdicción en Internet ya se han hecho valiosos estudios que arrojan luz sobre el camino. La evidencia recolectada demuestra que los gobiernos cada vez más están regulando las actividades y servicios de Internet para proteger los derechos de sus ciudadanos; en materia judicial, los jueces y tribunales están aplicando tanto reglas nuevas (online), como reglas tradicionales (offline) para resolver los conflictos de competencia y asumir o determinar la jurisdicción sobre los pleitos que se les presentan (Jiménez, 2015).

\section{La regulación de Internet y las tecnologías de la información y la comunicación en Colombia}

Como se sabe, Internet llega a Colombia a comienzos de los años noventa. En 1991 se conforma la primera red de universidades en Colombia llamada Runcol, compuesta por treinta universidades, el Instituto Colombiano de Fomento a la Educación Superior (hoy Instituto Colombiano para la Evaluación de la Educación) y la Empresa Colombiana de Telecomunicaciones (Telecom). Ese mismo año se logra la primera conexión entre la Universidad de Columbia (Estados Unidos) y la Universidad de los Andes (Colombia) a través de la red Bitnet de IBM.

Hubo muchas dificultades técnicas y económicas para que las universidades pudieran trabajar en red: la mayoría se conectaba mediante llamadas telefónicas de larga distancia, en horario esta- 
blecido y mediante el uso del módem; además, solo se ofrecía el servicio del correo electrónico. Hacia 1994 Runcol desaparece pero da origen a la Red Caldas, iniciativa del entonces Instituto Colombiano para el Desarrollo de la Ciencia y la Tecnología (Colciencias). Ese mismo año, se crea Cetcol una red mixta público-privada, y Telecom concibe el servicio Saitel que empezó a ofrecer servicios limitados como proveedor de Internet. En 1995 llega Compuserve, ya un IPS con todos los pergaminos, el cual marca el inicio de la popularización de Internet en el país (Cobos, 2010).

El desarrollo e implantación de estas nuevas tecnologías de la información presionaron para que la regulación e intervención estatal aparecieran hacia finales de los años ochenta del siglo pasado, generándose así, las primeras normas y disposiciones jurídicas sobre la materia. A continuación, y con un propósito meramente enunciativo, se hace una breve reseña cronológica de las principales normas que han venido surgiendo sobre informática, Internet y comercio electrónico.

- Ley 72/1989, otorga funciones de regulación del sector telemática e informática (derogada por ley 1341/2009).

- Decreto 1900/1990, establece que las telecomunicaciones son servicio público (teoría del servicio público, derogado por ley 1341/2009).

- Ley 37/1993, regula el servicio público de telefonía móvil celular.

- Ley 527/1999, reglamenta el comercio electrónico, el mensaje de datos y la firma electrónica (se dice que es una regulación avanzada en el contexto latinoamericano).

- Directiva presidencial 02/2000, trata sobre el gobierno en línea.

- Documento Conpes 3072/2000, constituye la Agenda de conectividad y se crea Intranet.

- Decreto $127 / 2001$, programa para desarrollo de las TIC y gobierno electrónico.

- Concepto Rad. 1376/2001 Concejo de Estado, Sala de Consulta Civil, sobre uso público del dominio ".co", concluye que el dominio ".co" debe ser administrado por el Estado colombiano.

- Ley 962/2005, sobre racionalización de trámites y procedimientos administrativos; establece el uso de medios tecnológicos o documentos electrónicos en las comunicaciones entre ciudadanos y la administración.

- Ley 1065/2006, sobre registro de dominios ".co".

- Resoluciones 1732/2007 y 1740/2007, sobre Comisión de Regulación de Telecomunicaciones.

- Decreto $1151 / 2008$, sobre gobierno en línea.

- Ley $1341 / 2009$, sobre la sociedad de la información y organización de las TIC, crea la Agencia Nacional de Espectro.

- Directiva presidencial 04/2012, estrategia "cero papel" y uso de medios electrónicos.

- Decreto 2364/2012, regula el artículo $7^{\circ}$ de la ley 527/1999 sobre la firma electrónica.

- Ley $1581 / 2012$ y decreto $1377 / 2012$, fundan el Régimen General de Protección de Datos Personales.

- Ley 1437/2011 (CPACA) y ley 1564/2012 (CGP), han incorporado al derecho procesal algunos aspectos de las TIC en lo referente a pruebas, notificaciones, audiencia en línea y la posibilidad del expediente electrónico (ver los artículos 53-64, 186 y 216 del CPACA y los artículos 103, 107 y 109 del CGP).

Entre las providencias más destacadas de la Corte Constitucional, se tienen las siguientes:

- Sentencia T-414 del 16 de junio de 1992 , M. P. Ciro Angarita Barón, primera sentencia en Colombia sobre protección de datos personales y habeas data, determina la prevalencia del derecho a la intimidad sobre el derecho a la información como consecuencia de la consagración de la dignidad humana como principio fundamental. 
- Sentencia C-662/2000, M. P. Fabio Morón Díaz, resuelve demanda de inconstitucionalidad sobre varios artículos de la ley 527/1999, encontrándolos ajustados a la Constitución.

- Sentencia C-831/2001, M. P. Álvaro Tafur Galvis, resuelve demanda de inconstitucionalidad del artículo 6 de la ley 527/1999 sobre la obligación establecida por una norma para que la información conste por escrito y este requisito se cumple con un mensaje de datos; se declaró su exequibilidad.

- Sentencia C-1147/2001, M. P. Manuel José Cepeda, se demanda el artículo 91 de la ley 633/2000 que dispone que las páginas web y sitios de Internet de origen colombiano cuya actividad sea financiera, comercial o prestación de servicios, deben inscribirse en el registro mercantil y suministrar a la Dirección de Impuestos y Aduanas Nacionales la información que esta requiera; se concluye que la norma no viola el derecho a la intimidad como solicitaba el actor.

- Sentencia C-981/2005, M. P. Clara Inés Vargas, se demandan algunos artículos de las leyes 788/2002 y 863/2003 y otros del Estatuto Tributario sobre información tributaria. El actor considera que estas normas deberían estar en una ley estatutaria y no en una ordinaria pues están relacionadas con el habeas data, y de las páginas web y de Internet se puede obtener información financiera y tributaria; se declara exequible.

- Sentencia C-1153/2005, M. P. Marco Gerardo Monroy, sobre control de constitucionalidad a proyecto de ley estatutaria sobre garantías electorales; se hace referencia a las encuestas por Internet contempladas en el proyecto y que la Corte avala.

- Sentencia T-208/2008, M. P. Clara Inés Vargas, el amparo se dirige contra el Instituto Colombiano de Crédito Educativo y Estudios Técnicos en el Exterior (Icetex) por negar un crédito educativo a una persona egresada de un colegio privado de Bogotá, quien alega que la convocatoria no lo restringía; Icetex argumentó que realizó una aclaración en su página web respecto de que los colegios privados deberían estar en concesión o en convenio con el distrito capital de donde provenían los fondos. Se niega el amparo.

- Sentencia T-013/2008, M. P. Marco Gerardo Monroy, establece alcance de la ley 962/2005 sobre derecho de petición, el cual puede hacerse mediante páginas web o escrito físico; el ciudadano escoge.

- Sentencia T-713/2010, M. P. María Victoria Calle, se resuelve revisión de tutela interpuesta contra la directora de un colegio que impuso matrícula condicional a un menor por unirse a un grupo de Facebook que apoyaba el cambio de rectora. Corte ampara los derechos fundamentales al buen nombre, a la honra, al debido proceso y ordena reformar el manual de convivencia.

- Sentencia C-058/2012, M. P. Humberto Sierra Porto, aquí se ampara el derecho de acceso a la justicia interpuesto por una menor quien había denunciado ante la Fiscalía a otra persona por injuria, al haber publicado en un correo de @hotmail.com una fotografía de la denunciante, argumentando que tenía sida. La denuncia ante la Fiscalía llevaba más de cinco años sin ningún avance.

- Sentencia T-260/2012, M. P. Humberto Sierra Porto, la tutela es presentada por la madre de una menor de cuatro años de edad, en contra del padre de la niña quien abrió una cuenta en Facebook a nombre de su hija, y la utiliza para desprestigiarla. El padre incumplió condiciones de Facebook (para abrir cuenta a nombre de otro se requiere autorización y tener al menos trece años de edad); la Corte ampara los derechos a la intimidad, al buen nombre y libre desarrollo de la personalidad, se ordenó al padre cancelar la cuenta y no abrir una nueva en otra red social. 
- Sentencia T-550/2012, M. P. Nilson Pinilla, tutela contra la Universidad del Rosario por expulsar a estudiante por emitir comentarios en Facebook en contra de los directivos. Se tutelan los derechos fundamentales de libre desarrollo de la personalidad, al trabajo y a expresar y difundir opiniones y pensamiento. La Corte confirmó fallo anterior negando el amparo al considerar que la universidad respetó el debido proceso y que el estudiante usó expresiones que no ampara la libertad de expresión.

- Sentencia T-643/2013, M. P. María Victoria Calle, la accionante había trabajado para empresa de masajes y había autorizado un estudio fotográfico; luego de finalizado el vínculo laboral pidió que retiraran las fotos de la página de Facebook sin éxito. Se solicita el amparo de los derechos a la intimidad, buen nombre, honra y dignidad humana. Corte establece que el carácter irrenunciable de los derechos fundamentales hace que la autorización de publicar las fotos, no implica una renuncia indefinida de su derecho. Se concede el amparo y se revocan los fallos anteriores que la negaban.

\section{Conclusiones}

La facilidad, rapidez y bajo costo de las comunicaciones en Internet han conducido a un aumento sin precedentes en las interacciones que cruzan fronteras de diferentes jurisdicciones y sistemas jurídicos, lo que suscita a su vez, el incremento de los conflictos jurídicos ocasionados en Internet. Aquí se presenta una paradoja: mientras las interacciones virtuales se amplían y se hacen más fáciles y a bajo costo, las soluciones a dichos conflictos parecen difíciles y costosas. Aquí es donde aparece la necesidad de realizar estudios e investigaciones sobe el derecho e Internet, que sustenten la posterior elaboración de regulación jurídica, la toma de decisiones gubernamentales y la labor hermenéutica en las providencias judiciales.

El hecho indiscutible es que estos conflictos jurídicos tienden a superar el territorio de una jurisdicción específica y se deben generar nuevos desarrollos normativos e interpretaciones jurídicas para que una determinada autoridad (internacional, nacional o incluso territorial), asuma la regulación, control y competencia en determinados casos. En este sentido, es menester que los abogados se aproximen al conocimiento de los aspectos tecnológicos de la comunicación virtual, para así entender, manejar y resolver los problemas jurídicos que surgen en el ambiente de Internet.

Los recientes desarrollos tecnológicos de las TIC e Internet como los nuevos aplicativos y dispositivos móviles, requieren una atención de parte de abogados y académicos sobre su impacto jurídico. La tendencia se dirige a un acrecentamiento de la portabilidad y conectividad en pequeños dispositivos que contienen una multiplicidad de tareas, servicios y aplicativos, con lo cual se hace más acentuado el carácter de portabilidad, rapidez, anonimato e independencia geográfica en las comunicaciones por Internet.

Los casos presentados en Colombia a la luz de las características de Internet, son apenas una pequeña muestra de las consecuencias de algunas de las particularidades de la comunicación por Internet y señalan una gran potencialidad para la investigación y práctica jurídicas. Se trata no solamente del incremento cada vez mayor de casos de Internet en los cuales se involucran los derecho a la intimidad, al buen nombre, a la libertad de expresión, los derechos de propiedad y de marcas, junto con los delitos informáticos y el comercio electrónico entre otros, sino del desarrollo de conocimiento jurídico para el tratamiento de esos temas.

Por otro lado, el importante desarrollo normativo y jurisprudencial en nuestro país demuestra que ya empezó todo un movimiento de incorporación de las TIC con el derecho, siendo Colombia un Estado que trata de mantenerse al día en estos asuntos; esto justifica aún más la necesidad de aportes desde la investigación y el conocimiento científico para que estos desarrollos jurídicos sean más adecuados y pertinentes a nuestra realidad 
social y cultura jurídica. En materia judicial, se observó un desarrollo muy focalizado con las disputas entre los derechos fundamentales a la intimidad y buen nombre, frente a los derechos de libertad de expresión y comunicación. Es en este campo, donde se espera contribuir en próximas investigaciones.

Por último, para el jurista y abogado, Internet debe ser visto más allá que un simple medio de comunicación del cual se podrían obtener beneficios en la práctica jurídica (tal es el caso de la informática jurídica). Si Internet ha cambiado nuestras vidas, debemos prestar mayor atención y escudriñar más detenidamente el fenómeno de la comunicación virtual, instantánea, crossborder y portable para elucidar sus consecuencias jurídicas. Sobre todo, en un campo de conocimientos y de práctica profesional como es el derecho, que tiende por su inercia normativa, a estar a la zaga de los acontecimientos.

\section{Referencias}

Alegsa. (s. f). Definición de portabilidad (informática). Disponible en: www.alegsa.com. arg/Dic/portabilidad

Cobos, T. (2010). Historia de Internet en el mundo y su llegada a Colombia. Disponible en: http://tanialu.co/2010/01/12/historiade-internet-en-el-mundo-y-su-llegada-acolombia/\#sthash.U6QpPac8.dpbs

Consejo de Estado. (2001). Sala de Consulta y Servicio Civil, radicado 1376, diciembre 11. M. P. César Hoyos Salazar.

Corte Constitucional. (2015). Sentencia T-277 de 12 de mayo. M. P. María Victoria Calle.

Corte Suprema de Justicia. (2010a). Sala de Casación Penal, caso Jerónimo A. Uribe, Auto rad. 33.474/2010.

Corte Suprema de Justicia. (2010b). Sala de Casación Penal, caso Centro Comercial Campanario, Auto rad. 34.564/2010.

De Lucca, N. (2012). Contratación informática y telemática. Bogotá: Temis.
Guerra, Y. (2012). Derecho y tecnología. Bogotá: Grupo Editorial Ibáñez.

Jiménez, W. G. (2015). Rules for offline and online in determining jurisdiction. Global overview and Colombian cases. International Law Revista Colombiana de Derecho Internacional, 26, pp. 13-62.

Jiménez, W. J. \& Lodder, A. (2015). Analyzing approaches to internet jurisdiction based on a model of harbors and the high seas. International Review of Law, Computers \& Technology, 29(2-3), pp. 266-282.

Kohl, U. (2010). Jurisdiction and the internet regulatory competence over online activity ( $2^{\mathrm{a}}$ ed.). Cambridge: Cambridge University Press.

Lodder, A. (2013). Ten commandments of Internet law revisited: basic principles for Internet lawyers. Information \& Communications Technology Law, 22(3), pp. 264-276. Disponible en: http://ssrn. com/abstract $=2343486$

Manrique, J. (2011). The assertion of judicial jurisdiction over cyber-torts. A comparative analysis. Revista de Derecho, Comunicaciones y Nuevas Tecnologías, 5, pp. 2-24.

Maquiavelo, N. (1993). El príncipe. Barcelona: Ediciones Altaya.

Peter, I. (s. f.) The history of email. Disponible en: http://www.nethistory.info/History $\% 20$ of $\% 20$ the $\% 20$ Internet/email.html

Quinto, D. (2003). Law of Internet disputes. Nueva York: Aspen Law Business.

Reidenberg, J. (2013). Internet jurisdiction: a survey of legal scholarship published in english and United States cases law. Fordham Center on Law and Information Policy -CLIP-. Working Paper 2309526. Disponible en: http://ssrn. com/abstract $=2309526$

Remolina, N. (2015). Zuckerberg, redes sociales digitales y el concepto de la Superintendencia de Industria y Comercio sobre el 
ámbito de aplicación de la ley colombiana de protección de datos. Observatorio Ciro Angarita Varón sobre Protección de Datos. Disponible en: http://habeasdatacolombia.uniandes.edu.co/? $\mathrm{p}=1718$

Superintendencia de Industria y Comercio. (2016). Radicación 14-218349-4-0, trámite 113, actuación 330 del 3 de marzo de 2016.

Svantesson, D. (2007). Private international law and the Internet. Rijn: Kluwer Law International.

Tato, N. (s. f.). El derecho informático como una nueva rama del derecho. Disponible en: http://www.nicolastato.com.ar/esp/ index.php?option=com_content\&view=ar ticle\&id=6:artderechoinformaticonuevara ma\&catid=10: categderechoinf\&Itemid=7

Tecnocosas. (s. f.). El correo electrónico: origen y funcionamiento. Disponible en: http:// www.tecnocosas.es/el-correo-electronicoorigen-y-funcionamiento/

Wang, F. (2010). Internet jurisdiction and choice of law: legal practices in the EU, US and China. Nueva York: Cambridge University Press. 